\title{
Review of: "Classical swine fever virus NS5A inhibits NF-KB signaling by targeting NEMO"
}

\author{
Kang-kang Guo
}

Potential competing interests: The author(s) declared that no potential competing interests exist.

The manuscript entitled "Classical swine fever virus NS5A inhibits NF-KB signaling by targeting NEMO" by Li and colleagues described an observation that the CSFV NS5A protein interacts NEMO. Mechanistically, NS5A mediated proteasomal degradation of NEMO by inducing K27-linked polyubiquitination of NEMO. Together, these finding proved that NS5A inhibits NF-KB signaling by facilitating NEMO degradation, which is a novel mechanism that CSFV inhibits host innate immunity. Overall, the idea is clear and concision and the manuscript is well written. The experiments were well-performed and appropriate control was included.

There are a number of concerns with this study which include the following:

1. The title and the conclusion of this study is that CSFV NS5A inhibits NF-kB signaling. However, little experiment was performed to measure the inhibition of NF-KB pathway, for instance the translocation of NF-KB(p65). In addition, using gene expression of IFNa to indicate NF-KB signaling activation or inhibition seems not enough. More experiments were need to demonstrate NS5A inhibits NF-kB signaling.

2. Fig 5B, different MOI of CSFV should be used to infect PK-15 cell and detect the degradation of NEMO.

3. The effect of NF-KB against CSFV infection should be measured. How about the viral load in NS5A overexpressed cells?

4. In some figure legend, "Evidence that..." is not necessary and it is better to delete it. 